\title{
The management of diabetic retinopathy in the public sector of eThekwini district of KwaZulu-Natal
}

\begin{tabular}{|c|c|}
\hline \multicolumn{2}{|c|}{$\begin{array}{l}\text { Authors: } \\
\text { Zaheera Abdool }{ }^{1} \\
\text { Kovin Naidoo } \\
\text { Linda Visser }\end{array}$} \\
\hline \multicolumn{2}{|c|}{$\begin{array}{l}\text { Affiliations: } \\
{ }^{1} \text { Department of Optometry, } \\
\text { Voortrekker Hospital, } \\
\text { South Africa }\end{array}$} \\
\hline \multicolumn{2}{|c|}{$\begin{array}{l}{ }^{2} \text { Brien Holden Vision Institute, } \\
\text { Durban, South Africa }\end{array}$} \\
\hline \multicolumn{2}{|c|}{$\begin{array}{l}{ }^{3} \text { Department of } \\
\text { Ophthalmology, University of } \\
\text { KwaZulu-Natal, South Africa }\end{array}$} \\
\hline \multicolumn{2}{|c|}{$\begin{array}{l}\text { Corresponding author: } \\
\text { Zaheera Abdool, } \\
\text { zaheeraa@webmail.co.za }\end{array}$} \\
\hline \multicolumn{2}{|c|}{$\begin{array}{l}\text { Dates: } \\
\text { Received: } 31 \text { Dec. } 2015 \\
\text { Accepted: } 17 \text { June } 2016 \\
\text { Published: } 19 \text { Aug. } 2016\end{array}$} \\
\hline \multicolumn{2}{|c|}{$\begin{array}{l}\text { How to cite this article: } \\
\text { Abdool Z, Naidoo K, Visser L. } \\
\text { The management of diabetic } \\
\text { retinopathy in the public } \\
\text { sector of eThekwini district of } \\
\text { KwaZulu-Natal. Afr Vision Eye } \\
\text { Health. 2016;75(1), a344. } \\
\text { http://dx.doi.org/10.4102/ } \\
\text { aveh.v75i1.344 }\end{array}$} \\
\hline \multicolumn{2}{|c|}{$\begin{array}{l}\text { Copyright: } \\
\text { (C) 2016. The Author( } \\
\text { Licensee: AOSIS. This } \\
\text { is licensed under the } \\
\text { Creative Commons } \\
\text { Attribution License. }\end{array}$} \\
\hline \multicolumn{2}{|l|}{ Read online: } \\
\hline 口أit: & $\begin{array}{l}\text { Scan this QR } \\
\text { code with your } \\
\text { smart phone or } \\
\text { mobile device } \\
\text { to read online. }\end{array}$ \\
\hline
\end{tabular}

Background: Estimates from the year 1990-2010 showed an increase in blindness and vision impairment (moderate or severe) because of diabetic retinopathy (DR) in Sub-Saharan Africa's sub-regions (central, eastern, southern and western Africa). ${ }^{1}$ The rate of DR in South Africa is expected to increase because of the lack of screening protocols and policies for the management of diabetic eye disease in the district health system of South Africa.

Aim: The purpose of this study was to determine the current role of healthcare practitioners (HCPs) towards managing DR in the eThekwini district of KwaZulu-Natal.

Method: A cross-sectional study was conducted, and questionnaires were distributed to a total of $104 \mathrm{HCPs}$ in public health institutions situated in the northern eThekwini district of KwaZulu-Natal. Clinics and community health centres (CHCs) were selected based on the assumption that primary healthcare nurses, medical officers (MOs) and ophthalmic nurses and/or optometrists practice at these institutions. The hospitals selected were the referral institutions for the selected clinics and CHCs. The questionnaires distributed included questions relating to diabetic patient registers, referrals to and from other HCPs, management of ocular complications, ocular screening methods, fundus examinations and involvement in screening programmes.

Results: Over a third of the ophthalmologists (35.3\%) indicated that DR was present at the initial examination in more than $50 \%$ of patients, though overall ophthalmologists reported loss of vision in at least one eye in fewer than $5 \%$ of patients on presentation. Less than half of the public sector general practitioners or MOs (40.6\%) conducted fundus examinations but $90.6 \%$ did not dilate pupils, although $71.9 \%$ had knowledge on the use of a direct ophthalmoscope. Only $40.6 \%$ of the MOs discussed the ocular complications of uncontrolled diabetes mellitus (DM) with patients and $62.5 \%$ encouraged regular eye examinations. Less than $50 \%$ of the MOs $(43.8 \%$ ) referred patients complaining of visual difficulties to optometrists and $9.4 \%$ referred to the ophthalmic nurses. Only $6.25 \%$ referred patients with DM needing further evaluation to ophthalmologists. Data from the optometrists were inconclusive because of the poor response rate of $5(20 \%)$. None of the ophthalmic nurses reported doing fundus photography or refractions. Two-thirds of the ophthalmic nurses were interested in training to properly grade DR.

Conclusion: The study established that there are key challenges in referral, training and practice in the management of DR. These need to be addressed in order to develop a comprehensive approach for the prevention and management of visual impairment and blindness because of DM.

\section{Introduction}

Diabetes mellitus (DM) is regarded as a global pandemic, currently having a worldwide prevalence of $8.5 \% .^{2}$ South Africa (SA) ranks as one of the top five countries in Africa, reflecting a 9.27\% prevalence of DM in the adult population (20-79 years). ${ }^{3}$ According to a demographic and health survey the highest prevalence of DM was found in KwaZulu-Natal (9\%), followed by the Western Cape (8.1\%), Gauteng (7.6\%), Eastern Cape (6.2\%), Northern Cape (5\%), Mpumalanga (4.8\%), Free State $(3.6 \%)$, Limpopo $(2.1 \%)$ and North West $(2.1 \%)$ provinces. ${ }^{4}$

Diabetes mellitus can lead to macrovascular (cardiovascular disease, cerebrovascular disease and peripheral vascular disease) and microvascular complications (nephropathy, neuropathy and retinopathy) which impact on the patients' quality of life, contributing to high morbidity and mortality rates. ${ }^{5}$ Regarded as the 6th leading cause of blindness globally, ${ }^{6}$ diabetic retinopathy (DR) is the most common cause of visual loss amongst working age (16-59 years 
for females and 16-64 years for males) individuals in developed countries. ${ }^{7}$ Diabetic retinopathy was recognised as a leading cause of blindness in developing countries more than a decade ago, ${ }^{8}$ and appears to be five times more prevalent amongst Type $2 \mathrm{DM}$ than Type 1 DM..$^{9}$ In subSaharan Africa (SSA) DR was found to be one of the six major causes of blindness. ${ }^{1}$ Amongst the latest DR prevalence studies conducted in three provinces in SA, KwaZulu-Natal reflected the highest prevalence of $40.3 \%{ }^{10}$ followed by Cape Town $(32.3 \%)^{11}$ and Gauteng $(22.8 \%){ }^{12}$

The duration of DM is an important predictor of DR development. ${ }^{13}$ Systemic illnesses associated with DR progression include severe hyperglycaemia, ${ }^{14}$ hypertension, ${ }^{15}$ nephropathy, ${ }^{16}$ hyperlipidaemia ${ }^{16,17,18}$ and HIV infections. ${ }^{19,20}$ Hyperlipidaemia is found to strongly correlate with the presence of DR $(75.4 \%)$, followed by anaemia $(42.5 \%)$, nephropathy (12.2\%) and neuropathy (9.3\%). ${ }^{13}$ However, the prevalence of severe DR is more likely associated with nephropathy and neuropathy. ${ }^{13}$ In spite of these numerous risk factors, research has clearly demonstrated that blindness due to DR is preventable with early diagnosis, minimisation of risk factors and timely photocoagulation where appropriate. $^{21}$

Laser photocoagulation therapy can reduce blindness due to DR by at least $60 \%,{ }^{22}$ but the problem is that vision loss due to DR may precede its diagnosis ${ }^{23}$ and sight threatening DR due to diabetic macula edema (DME) and proliferative DR (PDR) may not cause visual symptoms in their initial stages but may cause irreversible damage and blindness which might be too late to treat once detected. ${ }^{22}$ Also, surgical treatments are available to treat DR; however, these procedures could further complicate or reduce vision if instituted at an advanced stage in the diseases progression. ${ }^{23}$

Clinical practice guidelines for DR diagnosis have been adopted in various countries, but show variations in methods of examinations and also variations in health care practitioners (HCPs) involved in screening and diagnosing DR. ${ }^{21}$ Despite existing protocols for frequency of referral and grading criteria for DR in $\mathrm{SA}^{21}$ constraints in the district health system (DHS) have contributed to a lack of or poor implementation of DR screening programs. ${ }^{24,25}$

Since the progression of DR is dependent on the duration of $\mathrm{DM},{ }^{13}$ glycaemic control $^{26}$ and the presence of systemic complications $^{13}$, DM management is key to reducing DR progression. This situation therefore, demands a holistic, multidisciplinary team approach of HCPs to reduce blindness and other associated complications of DM which would increase DR progression. Each HCP has a specific role to play in the management of DM and DR and by understanding the skill and attitudes of these HCPs, an appropriate intervention strategy to manage DR in the DHS of SA can be developed. This study further intends to establish whether a DR screening program can be developed given the available resources in the eThekwini district of KwaZulu-Natal.

\section{Methodology}

A questionnaire-based, cross-sectional research design was used to gather information (roles, perceptions, awareness and attitudes) from primary healthcare nurses (PHC nurses), ophthalmic nurses, public sector general practitioners or medical officers (MOs), optometrists, ophthalmologists, clinical managers and operational managers (OMs) about the current status quo of managing DR in the eThekwini district of KwaZulu-Natal (Table 1). The study made use of both quantitative and qualitative methods to elicit the required information. After accessing the eThekwini district map, it was noted that there existed 58 clinics in the entire district. Because of the vast area and the long distance between various clinics, the northern region of eThekwini was considered for the research study. A list of all the clinics, community health centres (CHCs) and hospitals in the northern region of eThekwini district was accessed from the Department of Health website. Among the 15 listed clinics, 9 were undergoing policy reviews and renovations and converted to municipal clinics and therefore had to be excluded from the study. Six additional provincial clinics were then selected. However, during the questionnaire distribution phase, it was noted that two clinics merged with two other selected clinics and one converted to a $\mathrm{CHC}$, totalling to 12 clinics. Responses from participants were received from 11 out of the 12 clinics, 6 out of the $6 \mathrm{CHCs}$ and 4 out of the 4 hospitals. The inclusion criteria for participants were HCPs involved in the clinical management of DM and diabetic eye complications.

The exclusion criteria were HCPs who were not qualified to manage diabetic eye complications. The protocol was submitted to the University of KwaZulu-Natal's School of Health Sciences Faculty Review Committee and then to the Biomedical Research and Ethics Committee. Ethical clearance was granted before commencement of the study.

Permission to conduct the research study at the various hospitals, clinics and CHCs was obtained from the Department of Health KwaZulu-Natal and the respective Heads of Institutions of selected health institutions. The research objectives were communicated in writing in both English and isiZulu. Informed consent was obtained from all participants. Data were analysed using Stata, version 11. Descriptive statistics summarised the key opinions and recommendations. Inferential results were analysed using crosstabs. Fisher's exact test one was used to see if

TABLE 1: Categories and number of participants to whom questionnaires were provided.

\begin{tabular}{lccc}
\hline Categories & $\begin{array}{c}\text { Number of participants } \\
\text { who completed } \\
\text { questionnaires }\end{array}$ & $\begin{array}{c}\text { Total number of } \\
\text { invited } \\
\text { participants }\end{array}$ & $\begin{array}{c}\text { Response } \\
\text { rate (\%) }\end{array}$ \\
\hline Primary healthcare nurses & 42 & 89 & 47.2 \\
Ophthalmic nurses & 5 & 14 & 35.7 \\
Medical officers & 30 & 52 & 57.7 \\
Optometrists & 1 & 5 & 20.0 \\
Ophthalmologists & 17 & 17 & 100.0 \\
$\begin{array}{l}\text { Clinical managers and } \\
\text { Operational managers }\end{array}$ & 9 & 15 & 60.0 \\
\hline Total & $\mathbf{1 0 4}$ & $\mathbf{1 9 2}$ & $\mathbf{5 4 . 1}$ \\
\hline
\end{tabular}


the variables were related when the data cells were less than five.

\section{Results}

\section{Primary health care nurses}

Almost a third of the PHC nurses (31.0\%) estimated seeing more than 100 patients with diabetes per month. Nearly a quarter of the referrals (23.8\%) were from traditional healers, and other referrals received (46.7\%) were from local clinics, $\mathrm{CHCs}$, general practitioners, churches, hospitals and selfreferrals (Figure 1).

Forty-three percent of PHC nurses referred patients with DM who complained of ocular complications to ophthalmic nurses only and $11.9 \%$ referred patients to optometrists. However, $38.1 \%$ responded that they referred patients to both cadres (Figure 2).

The PHC nurses indicated having a 100\% role in taking case history, and the tests least conducted included pupil dilation and fundus examination (Figure 3).

Two-thirds of the PHC nurses were willing to support an outreach programme for DR. Reasons for not being able to

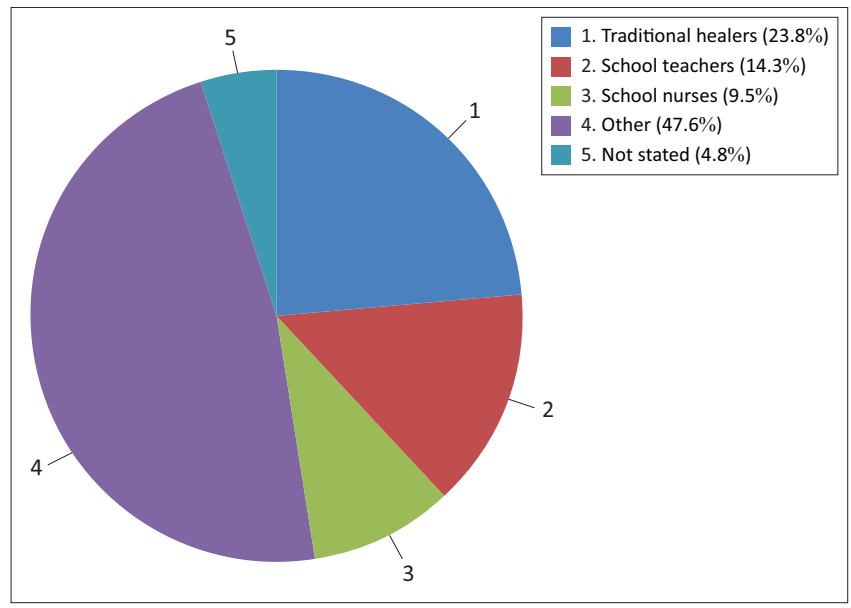

FIGURE 1: Percentage distribution of referrals to primary healthcare nurses.

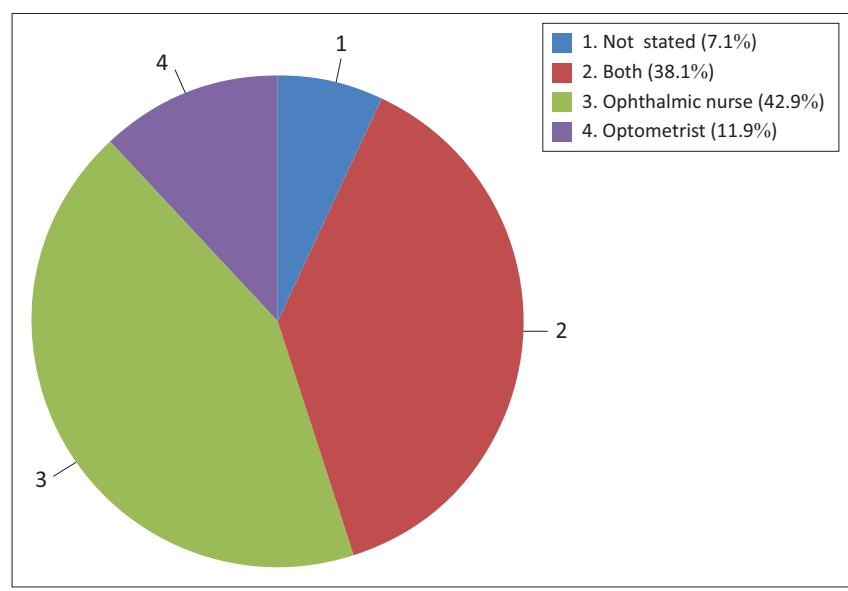

FIGURE 2: Percentage referrals from primary healthcare nurses to ophthalmic nurses and optometrists. support a DR screening programme included being involved in other screening programmes $(4.8 \%)$, having other responsibilities $(9.5 \%)$, staff shortage $(4.8 \%)$ and having insufficient qualifications (2.4\%).

\section{Ophthalmic nurses}

Two of the five ophthalmic nurses estimated seeing more than 100 patients with DM per month. Ophthalmic nurses reported receiving referrals uniformly from PHC nurses (1), optometrists (1), general practitioners (1) and traditional healers (1). Four of the five ophthalmic nurses did not state having a role in examining the fundus even though two stated using a direct ophthalmoscope when questioned about which instrument they used to view the fundus (Table 2).

Four of the five ophthalmic nurses indicated having a role in taking case history, and only three reported dilating pupils (Figure 4). Four did not respond when questioned about conducting refraction and examining the fundus.

Three screened for cataract, two for glaucoma, two for DR and one reported screening for other eye conditions such as squints. Only one ophthalmic nurse was familiar with grading DR, three were not and two did not respond. None of the respondents indicated the classification system used. Four of the ophthalmic nurses were interested in further training to detect and grade retinal lesions. Four of the ophthalmic nurses conduct screening as part of their job description. Four of the five ophthalmic nurses were willing to support an outreach programme for DR.

\section{Optometrists}

The response rate of optometrists in the public sector was very low as only one optometrist eventually participated in

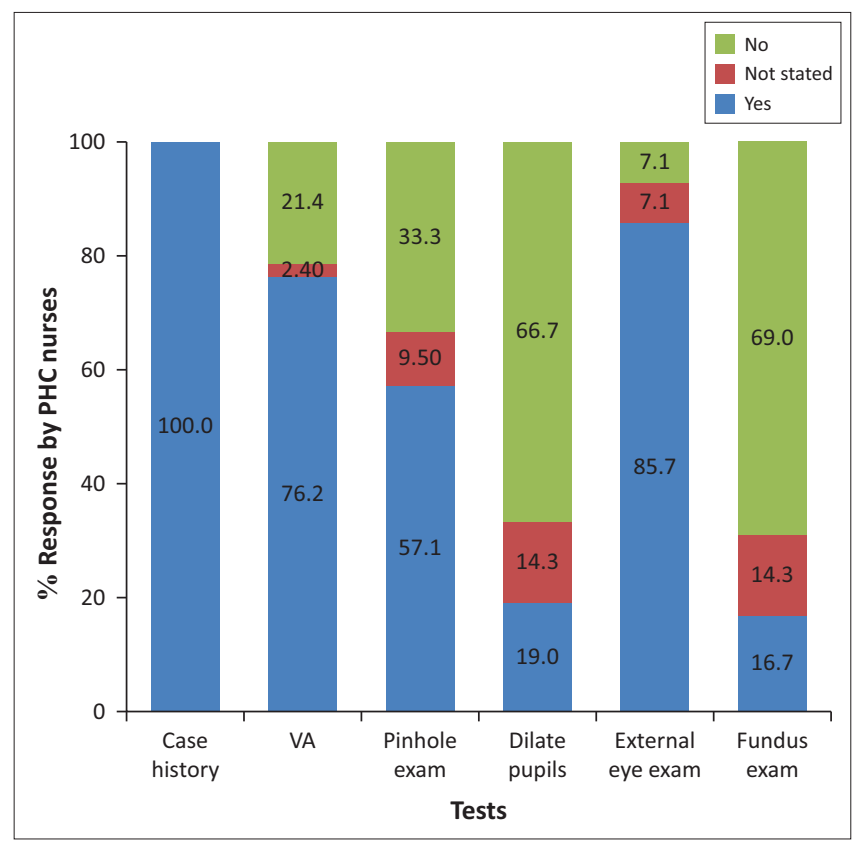

FIGURE 3: The self-estimated (in percentages) role of primary healthcare nurses in conducting ocular tests. 
TABLE 2: Percentage distribution of equipment used by primary healthcare nurses and ophthalmic nurses to perform fundoscopy.

\begin{tabular}{|c|c|c|c|c|}
\hline \multirow{2}{*}{$\begin{array}{l}\text { Equipment used by primary } \\
\text { healthcare nurses to perform } \\
\text { fundoscopy }\end{array}$} & \multicolumn{2}{|c|}{ Primary healthcare nurses $(n=42)$} & \multicolumn{2}{|c|}{ Ophthalmic nurses $(n=5)$} \\
\hline & $n$ & $\%$ & $n$ & $\%$ \\
\hline Direct ophthalmoscope & 7 & 16.7 & 2 & 40 \\
\hline Fundus biomicroscopy & 0 & 0.0 & 0 & 0 \\
\hline Fundus camera & 3 & 7.2 & 0 & 0 \\
\hline
\end{tabular}

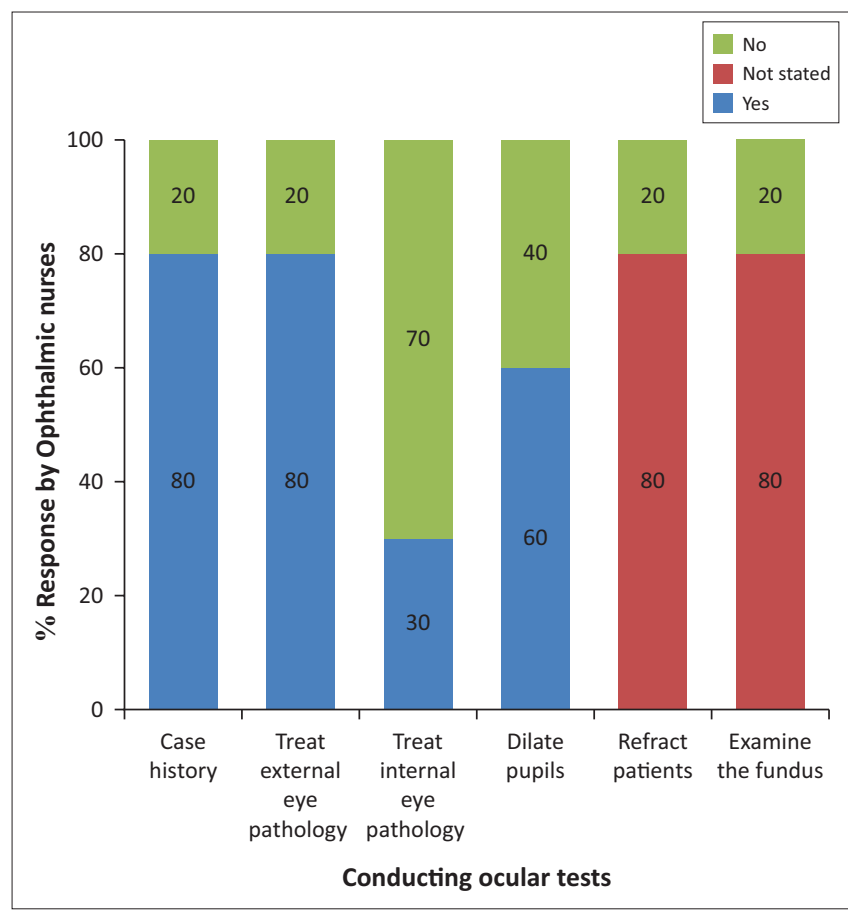

FIGURE 4: Percentage distribution of the role of ophthalmic nurses in conducting ocular tests.

the study. Because of this low response rate, private sector optometrists were also invited to participate. The questionnaires were distributed through a unique email system to all the members of the South African Optometric Association in Kwazulu-Natal. Because this database was confidential, information regarding name, number and contact details of optometrists could not be accessed. However, only four responses were received and the data were therefore not presented here.

\section{Public sector general practitioners or medical officers}

Almost half the participating MOs (46.9\%) estimated seeing more than 100 patients with DM per month. Most of the MOs $(81.3 \%)$ received referrals from PHC nurses. They also received other referrals (21.9\%) from private and public general practitioners, physiotherapists, self-referrals and from the outpatient departments. Figure 5 shows the distribution of the referrals received and respondents could give multiple responses; therefore, the overall percentage is more than $100 \%$.

The majority of MOs (68.8\%) indicated that their case history occasionally included questions about visual difficulties. Visual acuity (VA) was taken on all patients with DM complaining of visual difficulties by $15.6 \%$ of the MOs. About

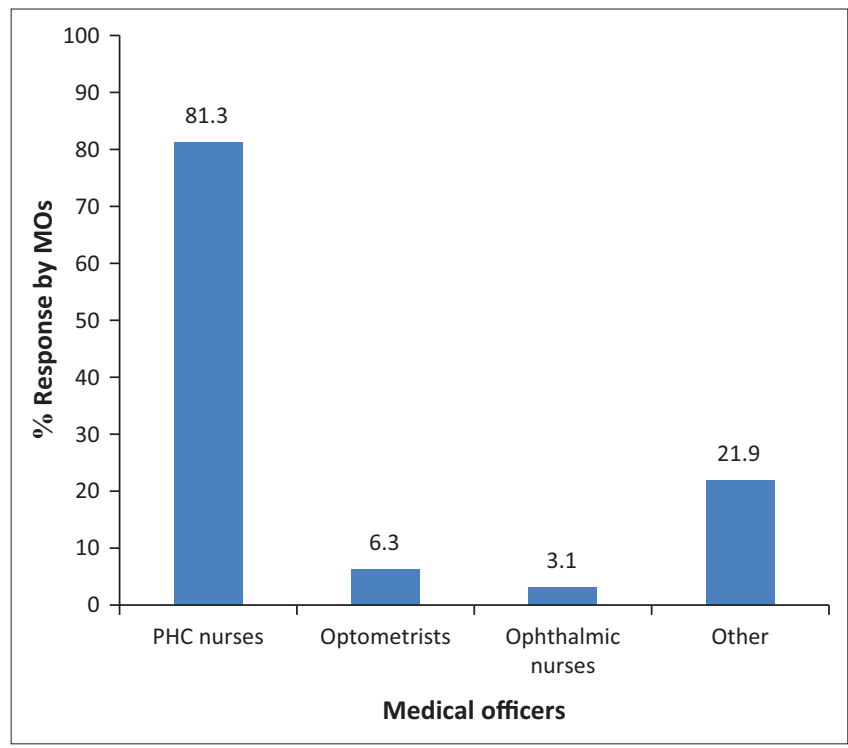

FIGURE 5: Percentage distribution of referrals to medical officers.

$44 \%$ referred patients who complained of visual difficulties to optometrists, less than $10 \%$ referred to ophthalmic nurses and $6.3 \%$ referred to ophthalmologists. Less than 50\% (46.9\%) examined the fundus and more than $90 \%$ did not dilate pupils. Forty percent always discussed the ocular complications of uncontrolled DM with their patients and $62.5 \%$ encouraged regular eye examinations. Seventy-two percent reported using a direct ophthalmoscope to view the fundus and only $9.9 \%$ had the opportunity to use a fundus camera. About $69 \%$ were willing to support a screening programme for DR. Twenty-five percent were not interested in supporting a programme for DR because of a heavy workload, and having no time, having staff shortages, being session doctors and not being qualified to screen for DR.

\section{Ophthalmologists}

Half the sampled participant ophthalmologists estimated seeing 20-50 patients with DM per month. A third (35.3\%) indicated that more than $50 \%$ of patients with diabetes who were referred had DR on their first visit and nearly half of the ophthalmologists (47.1\%) indicated that less than $5 \%$ of these patients had already lost vision in one eye because of DR (Table 3, Figure 6).

Forty percent of the ophthalmologists reported receiving more referrals from optometrists than MOs in the percentage category of $11 \%-20 \%$ referrals; however, more referrals were received from MOs than optometrists in the percentage category referrals of less than 5 and greater than 20 as seen in the graph in Figure 7. 
TABLE 3: Percentage of patients presenting with diabetic retinopathy on first visit and percentage who have lost vision in one eye because of diabetic retinopathy

\begin{tabular}{|c|c|c|c|c|}
\hline \multirow[t]{2}{*}{$\begin{array}{l}\text { Percentage of patients reported } \\
\text { by ophthalmologists }(\%)\end{array}$} & \multicolumn{2}{|c|}{$\begin{array}{c}\text { Ophthalmologists }(\%) \text { reporting patients presenting } \\
\text { with diabetic retinopathy at first visit }\end{array}$} & \multicolumn{2}{|c|}{$\begin{array}{l}\text { Ophthalmologists (\%) reporting patients having vision } \\
\text { loss because of diabetic retinopathy at first visit }\end{array}$} \\
\hline & $n$ & $\%$ & $n$ & $\%$ \\
\hline$<5$ & 3 & 17.6 & 8 & 47.1 \\
\hline $11-20$ & 3 & 17.6 & 2 & 11.8 \\
\hline $21-50$ & 1 & 5.9 & 2 & 11.8 \\
\hline$>50$ & 6 & 35.3 & 2 & 11.8 \\
\hline
\end{tabular}

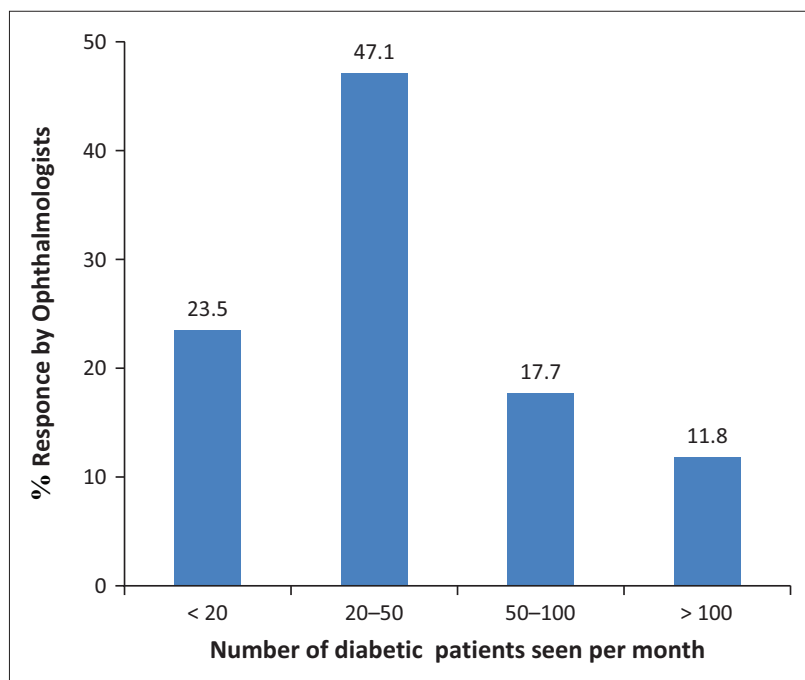

FIGURE 6: Number of patients seen per month by ophthalmologists.

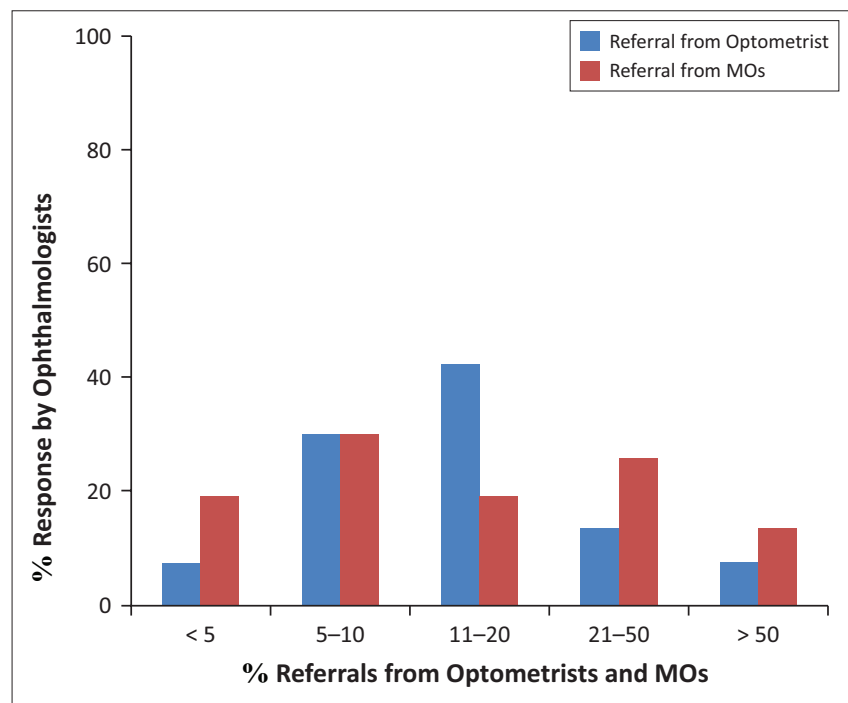

FIGURE 7: Referral percentages from optometrists and medical officers to ophthalmologists.

Approximately $30 \%$ of the ophthalmologists indicated that $5 \%-10 \%$ of patients with DM who were referred to them by HCPs already had manifestations beyond non-proliferative DR as shown in Figure 8.

Referrals for most ophthalmologists were from optometrists and physicians (91.4\%). These referrals were considered most of the time appropriate by $70.6 \%$ of the ophthalmologists. In addition to the types of referrals, ophthalmologists indicated receiving referrals from neurologists $(17.6 \%)$, endocrinologists (29.4\%), and nephrologists $(11.8 \%)$ in a multiple response

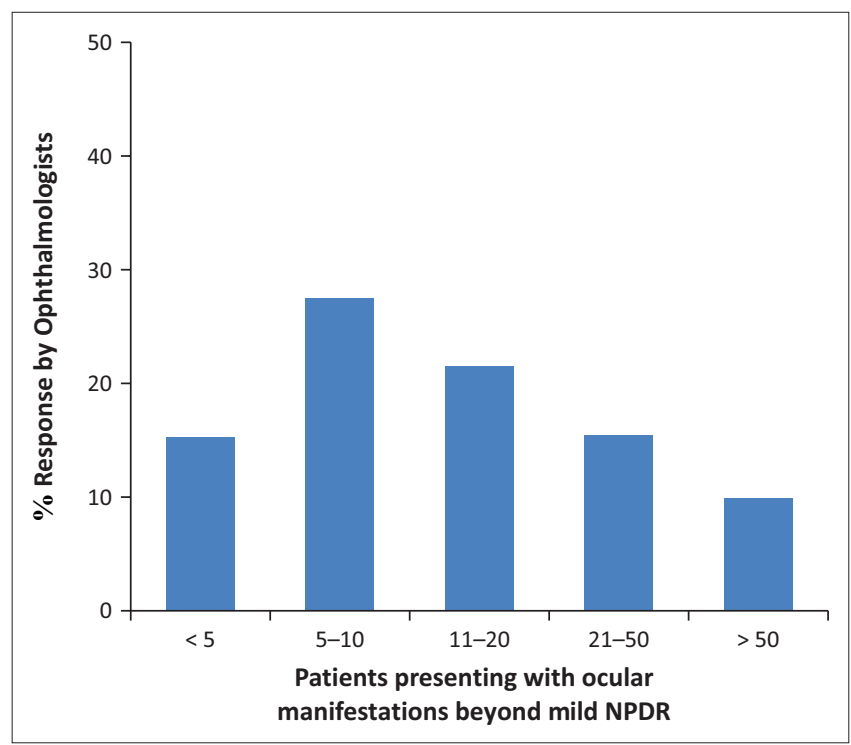

FIGURE 8: Percentage response by ophthalmologists indicating patients presenting with ocular manifestations beyond mild non-proliferative diabetic retinopathy.

question. Results of most referrals received are depicted in Figure 9. Less than a quarter of ophthalmologists (23.5\%) stated that the DR status of all patients with diabetes referred to them was most of the time classified.

Most of the ophthalmologists $(82.4 \%)$ indicated that the fundus camera is the most suitable instrument to detect DR at a PHC level followed by the ophthalmoscope (58.8\%) and the slit lamp (17.7\%). The responses received from the 17 ophthalmologists regarding the most common to least common eye conditions seen in daily practice in patients with DM are reflected in Figure 10. The most common eye condition was assigned a weight of 6 and the least common was assigned a weight of 1 . Thereafter, a weighted average was calculated and the results are presented in Figure 10.

\section{Clinical managers (medical managers at hospitals) and operational managers (nursing managers at community health centres and clinics)}

Eight of the nine managers reported DM to be a very important problem in the eThekwini district of KwaZuluNatal, and four reported diabetic eye disease to be a very important problem. Six reported not having specific policies and protocols on diabetic eye disease management. All the reporting managers indicated that they required further training on diabetic eye disease management and also suggested that the PHC nurses as well as the MOs be included in the training (Figure 11). 


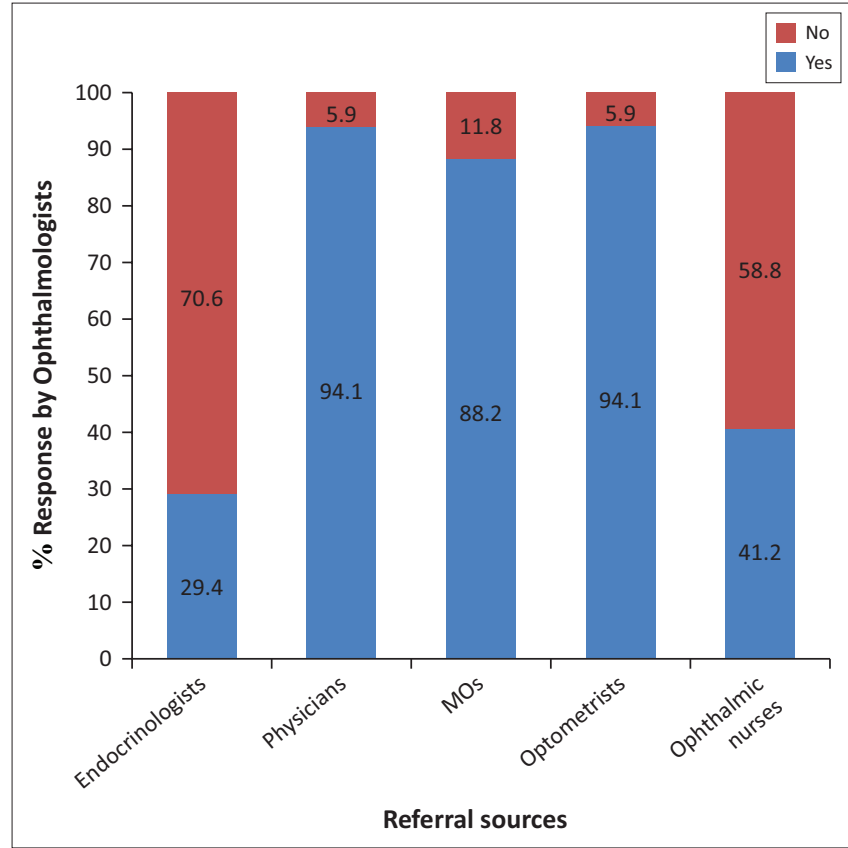

FIGURE 9: Referral sources to ophthalmologists.

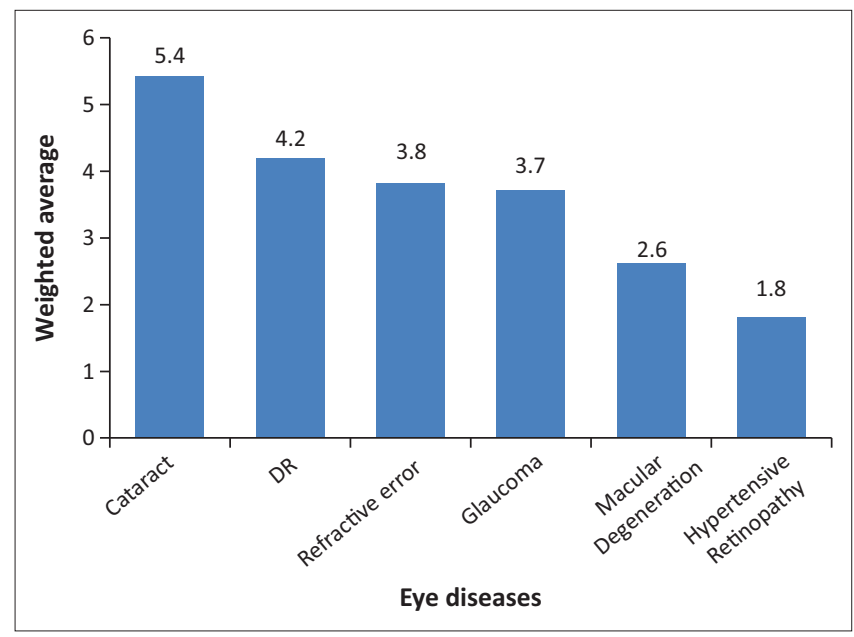

FIGURE 10: Response by ophthalmologists on rating common eye diseases seen in patients with diabetes.

Seven of the nine clinical managers and OMs reported having eye screening programmes in the district, and eight of the nine reported having referral sites within the district for the management of ocular complications because of DM. When questioned about the availability of posts for PHC nurses, ophthalmic nurses, optometrists and MOs, four managers indicated that the posts for the above professionals were filled, three reported the posts vacant and two did not respond. Only three managers felt that a diabetic eye screening programme was possible given the current number of PHC and ophthalmic nurses in the district. Six indicated that the health district will be able to create more posts should further human resources be required.

The most common existing barrier to the implementation of a diabetic eye screening programme in the opinion of the managers was the lack of equipment (cited by eight), and the

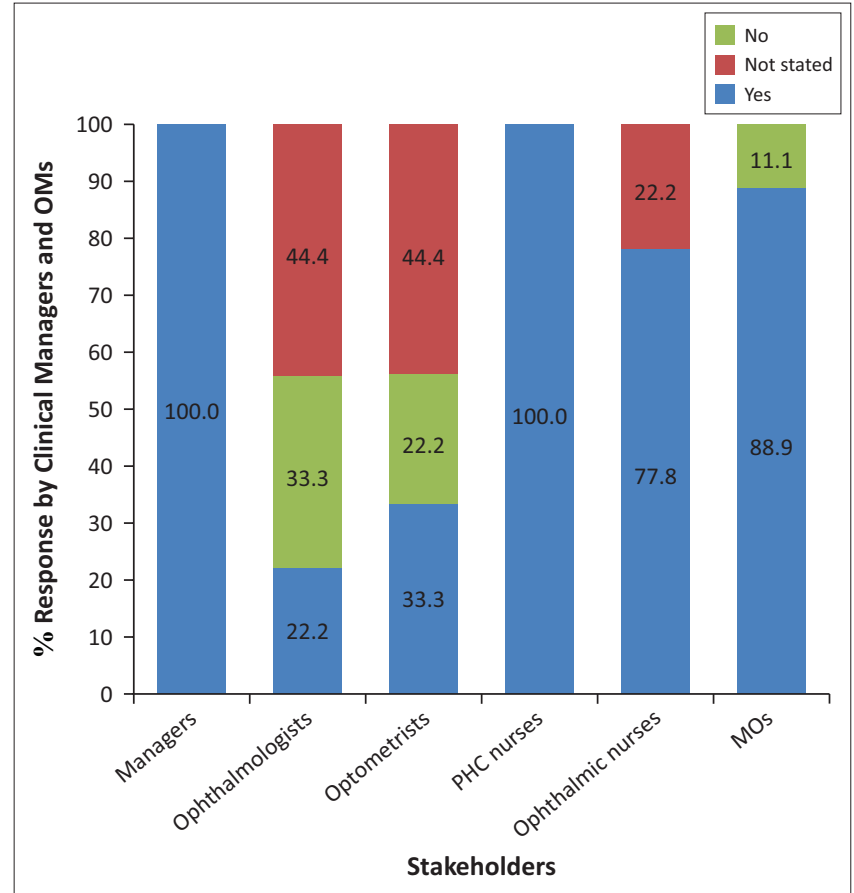

FIGURE 11: Percentage distribution for stakeholders requiring further training on diabetic eye disease management.

lack of interest by HCPs (cited by one) was the least common existing barrier reported (Figure 12).

\section{Discussion}

\section{Primary healthcare nurses}

The PHC nurses play an important role in building a network for referral and screening at the community level. They bridge the divide between community and other healthcare workers as they refer patients with DM complaining of ocular complications to ophthalmic nurses and optometrists. They are mainly responsible for taking case history, screening vitals and providing patient education on the management of systemic complications. However, some were found to be involved in ocular screening and were familiar with some optometric evaluation procedures. Their knowledge regarding the use of a fundus camera, indirect ophthalmoscope and slit lamp was limited. However, their knowledge on the use of a direct ophthalmoscope is a good basis in which to build a sustainable and comprehensive eye health programme. With regard to other ocular screening tests such as taking VAs and pinhole test, they have good basic skills. Screening is mainly conducted at the clinics. Their willingness to support a DR outreach programme is encouraging; however, human resource issues and training create barriers. Two-thirds of the PHC nurses were willing to support an outreach programme for DR, which is impressive; however, a policy for the management of DR needs to be developed and implemented; targeted outreach programmes need to be planned to screen patients with DM; and training programmes for DR detection must be introduced.

\section{Ophthalmic nurses}

All ophthalmic nurses are based at clinics. They are an important bridge between the PHC nurses and other 


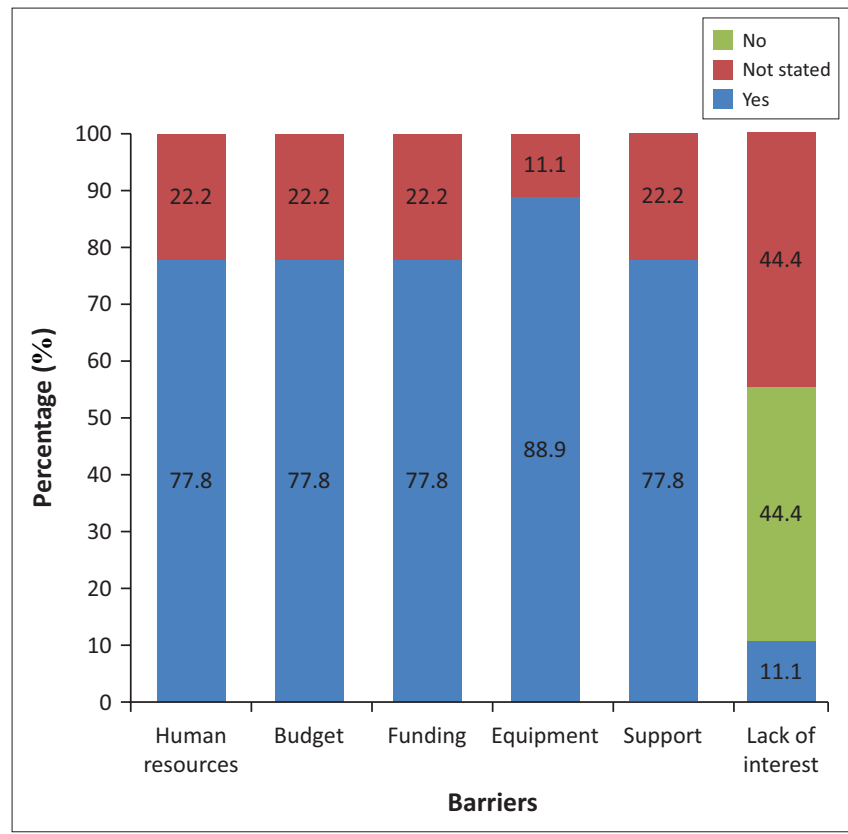

FIGURE 12: Percentage distributions on existing barriers to implementing a diabetic eye screening programme.

professionals such as optometrists and ophthalmologists. Their proximity to the communities makes them an ideal cadre in the diabetic eye care team. In this study, none of the ophthalmic nurses conducted fundus photography, as no fundus cameras were available in the workplace. However, two out of five ophthalmic nurses used a direct ophthalmoscope. The study established that the ophthalmic nurses spent less time than the PHC nurses in taking case history, testing blood glucose, taking urine samples and giving patient education. More time was spent in taking VA and dilating pupils. The training received by the ophthalmic nurses did not sufficiently cover diabetic eye diseases, and their self-reported knowledge on DR grading was poor. Although ophthalmic nurses did not screen for DR, they did refer newly diagnosed DM patients to optometrists and ophthalmologists. The referral was done after taking VAs and dilating pupils. Interest has been shown in training to detect and grade retinal lesions. Ophthalmic nurses are willing to support an outreach programme for DR. The current diploma curriculum available for nurses qualifies ophthalmic nurses to perform a VA assessment, take a case history, perform dilated eye examinations, manage chronic eye conditions and make effective referrals. ${ }^{27} \mathrm{~A}$ concern raised by one of the ophthalmologists regarding implementation of eye screening programmes for ophthalmic nurses was that slit lamps were not always available and that ophthalmic nurses should learn to examine the anterior eye segment with a torch. ${ }^{27}$ This is indicative of ophthalmic nurses being unable to utilise their specialised skills. The clinical environment should be receptive and encourage the expansion of nursing roles. This can be achieved if attitudes, policies and resource allocation in the workplace can be modified.

\section{Optometrists}

The role of optometrists in general includes conducting both external and internal eye examinations; however, having insufficient training to detect and classify DR prevents optometrists from appropriately screening, grading, referring and recalling patients who need to be monitored. There is a need for more optometrists to be employed in the public sector especially because optometrists are graduating with diagnostic skills and can therefore conduct a comprehensive fundus evaluation. This would in turn reduce the burden on ophthalmologists and provide support for their pre- and post-laser treatment by monitoring DR progression. Joint continuing education workshops and lectures regarding the management of eye care patients in the public sector should be explored. There is a need to integrate private and public sector activity. Information from the public sector on district protocols with regard to the management of eye care should be communicated to the private optometrists so that they advise and manage patients appropriately. There is also a need to evaluate the education and training at optometry institutions and incorporate a greater emphasis on DR. Optometrists could be utilised in conducting training programmes for the ophthalmic nurses and PHC nurses and also in the development of the appropriate health promotion material. However, for optometrists to make a significant contribution, greater integration into the public sector eye health team needs to happen.

\section{Medical officers}

A study has shown that MOs find methods of DR screening such as dilated indirect ophthalmoscopy and fundus photography to be time consuming and furthermore lack the training. ${ }^{28}$ It is also established that most MOs do not generally stay at an institution beyond 6 months..$^{11,29}$ It is also found that MOs fear dilating patients' eyes because of the risk of precipitating angle closure glaucoma. ${ }^{30}$ Other concerns include the lack of dilating drops in practice, the lack of confidence in detecting DR changes, concerns regarding time taken and the lack of ophthalmoscopes in practice. ${ }^{30,31}$

In this study, it was established that MOs often rotate between clinics and CHCs. Most referrals received were from the PHC nurses. Patients complaining of visual difficulties were more often referred by MOs to optometrists than to ophthalmic nurses. Not all MOs discussed the ocular complications of uncontrolled DM with their patients. Among those who examined the fundus, all used a direct ophthalmoscope. Pupil dilation was not done prior to fundus examination. Reasons for this were not established.

Most were in support of a DR screening programme, despite being short-staffed and having insufficient training in the field. There is a need for protocols to be developed regarding the management of ocular complications of DM for MOs, and regulations have to be in place to practice such protocols and systems for monitoring and evaluation of practices must be implemented.

\section{Ophthalmologists}

On average, 20-50 patients with DM are examined per month per ophthalmologist. Ophthalmologists received the majority 
of their referrals from physicians, optometrists and general practitioners. The DR status was occasionally classified on referral. These referrals were most of the time appropriate but not accurately graded. This suggests a lack of education in grading DR by the referral practitioners, probably because of the lack of a standard grading classification system for DR in South Africa. Hospitals that have developed and adopted standard grading classifications have had success rates in recruiting various personnel (medical and non-medical) to detect, grade and classify DR. ${ }^{32}$ Current grading classifications used in South Africa include the Early Treatment Diabetic Retinopathy Studies classification system mainly used by ophthalmologists ${ }^{25}$ and the UK National Screening grading classification system used by trained photographers. ${ }^{11,33}$ Ophthalmologists in KwaZulu-Natal prefer the Scottish grading classification system (Visser L., pers. comm., 09 September 2013), which is slightly more aligned with the International DR Classification Scale. ${ }^{34}$ The implication is that classification systems used are based on training received.Ithas been established thatophthalmologists who detected, graded and classified DR for more than 2-4 hours per day found this task tiring and visually demanding. ${ }^{11}$

Ophthalmologists in this study rated DR as the second most common eye disease seen in daily practice. Therefore, training is required for PHC workers to detect, grade and classify DRappropriately before referring to ophthalmologists. The introduction of training programmes will result in more appropriate referrals to ophthalmologists and patients not needing surgical intervention yet will be kept out of busy clinics (especially in the public sector) and will be monitored by trained graders.

\section{Clinical managers and operational managers}

$\mathrm{DM}$ and DR are both considered important issues in the district by all clinical managers. At present, there are no specific policies and protocols for the management of diabetic eye disease in the district. The recommendation made by all nine participating clinical managers and OMs is that they be trained on the management of diabetic eye disease. They also felt that PHC nurses, ophthalmic nurses as well as MOs required the training. A high proportion of clinical managers and OMs did not state whether further training is required for ophthalmologists, optometrists and ophthalmic nurses. This may be a reflection of the distinctive separation of the ophthalmic profession from other clinical departments. Furthermore, clinical managers and OMs may not be familiar with the functional aspects of the ophthalmic department. A high proportion did not respond to whether the lack of interest posed as a barrier to implementing a diabetic eye screening programme, and this may be because of a lack of understanding or disinterest in participation. The barriers reported to impact on the implementation of a diabetic eye screening programme included a lack of equipment, reduced number of staff, reduced budget, lack of funding and a lack of support. The engagement of key managers in the Department of Health is important to address these barriers.
The National Guidelines for DR were set in 2002 by the Department of Health and were intended for clinical practitioners, health service co-ordinators and primary eye care workers. ${ }^{21}$ Despite the existing protocol for frequency of referral and grading criteria for DR, constraints in the district health system contributed to a lack of or poor implementation of DR screening programmes. Constraints include a lack of human resources where there were inadequate specialists to address the needs of patients with DM and poor photographer turnover to operate fundus cameras and grade DR lesions. ${ }^{25}$ Other constraints included the lack of effective prevention strategies for DR where patients with DM had poor access to HCPs such as dieticians and psychologists who could assist with the non-pharmacological aspects of glycaemic control. ${ }^{24}$ Furthermore, inadequate financial resources lead to the lack of funds to develop treatment centres for patients with DM. ${ }^{25}$

At present in the South African district health system, team members responsible for DM management and its associated complications extend from the community to the CHCs and clinics (primary levels of care) to the district hospitals (secondary level of care) and finally to the provincial or regional hospitals (tertiary level of care). Team members may be present at primary level where the principal goal is to reduce the incidence of preventable DR by increasing awareness on diabetic eye complications and screening patients with DM. ${ }^{35}$ At the secondary level, DR may be treated using laser therapy and other medical treatment. ${ }^{14}$ Tertiary level includes the provision of vitreo-retinal surgery and advanced investigations for DR such as fluorescein angiography, photography-scan ultrasonography and the use of lasers to treat DR. ${ }^{35}$ Health education on DM and diabetic eye disease is required at all levels of care ${ }^{35,36}$ as well as human resource development and research. ${ }^{14}$ PHC nurses, ophthalmic nurses, general practitioners, optometrists and ophthalmologists are required at each level of care and have specific roles to play in DR management, which includes its prevention, detection, grading, referring and monitoring.

\section{Limitations of the study Small sample size of optometrists}

None of the clinics selected for the study were staffed with optometrists. The four hospital institutions selected were each staffed with one optometrist. The two optometrists employed at the two $\mathrm{CHC}$ were appointed as sessional optometrists rotating services to some CHCs.

\section{Poor response rate by optometrists}

During the questionnaire distribution phase, it was noted that there was a general lack of optometrists in public practice. Therefore, private sector optometrists were included to participate in the study. Private and public sector optometrists were informed about the study on two occasions via a unique email distribution system through the South African Optometric Association. Optometrists listed in the Yellow Pages were randomly selected and telephonically contacted and emailed. Only two responded from the unique 
email system, three responded from the telephonic enquiry and two from the direct email. From the direct email, both refused participation. From the public sector, only one participant from a tertiary hospital completed the questionnaire. Participation in this study was voluntary, and despite efforts to encourage participation which also increased study expenses, poor compliancy was shown.

A greater response or participation rate by the optometrists would enable us to generalise the results to the optometrists practicing in the eThekwini district of KwaZulu-Natal. The alarmingly low response may indicate a general lack of knowledge that optometrists perceive to have about DR, which would emphasise the priority that needs to be given to increasing the awareness, education and training regarding diabetic eye disease.

\section{Conclusion}

The evidence indicates that there are many gaps in the management of diabetic eye disease in the public sector both at the level of the skills and training of specific cadres and also at the level of the integration of the various cadres into a team that complements each other's efforts.

Appropriate policies for primary and secondary prevention of DR need to be developed at a provincial level to ensure that the eagerness by managers to support diabetic eye care programmes is galvanised. Primary prevention for DR should involve the introduction of patient education programmes and public awareness campaigns that would emphasise the importance of systemic control of DM to prevent visual loss because of DR. This task should be allocated to community health workers, school health nurses, traditional healers, PHC nurses and general practitioners or MOs. Secondary prevention will involve the screening of all patients presenting with DM and detecting, classifying and referring DR and associated eye complications to ophthalmologists or trained MOs for laser treatment. The screening of DR and monitoring of pre-proliferative DR stages should be done by trained PHC nurses, ophthalmic nurses, optometrists, and general practitioners or MOs. Resources required for the primary and secondary prevention of DR will include infrastructure such as a dedicated DM patient care facility, the recruitment of staff with clear job descriptions, the introduction of DR training programmes, the provision of equipment to screen for and treat DR and a quality assurance system to evaluate the performance of graders. In the United Kingdom, the National Screening Committee has implemented a successful DR screening programme in an effort to effectively combat blindness because of DR. Training is provided to interested candidates such as photographers and educators and is not limited to only HCPs. Appropriate policies will allow for budgetary allocation and the necessary human resource deployment. In the absence of these, district managers will be constrained in their capacity to prioritise diabetic eye disease.

\section{Acknowledgements Competing interests}

The authors declare that they have no financial or personal relationships which may have inappropriately influenced them in writing this article.

\section{Authors' contributions}

Z.A. was project leader, K.N. and L.V. (postgraduate supervisors) reviewed content of questionnaires for applicability, construct validity and reliability and assisted in data analysis.

\section{References}

1. Naidoo K, Gichuhi S, Basáñez MG, et al. Prevalence and causes of vision loss in sub-Saharan Africa: 1990-2010. Br J Ophthalmol. 2014;98(5):612-618. http:// dx.doi.org/10.1136/bjophthalmol-2013-304081

2. World Health Organization. Global Report on Diabetes. Geneva, Switzerland 2016. Page 6. [cited 2016 Apr. 11]. Available from http://apps.who.int/iris/ bitstream/10665/204871/1/1/9789241565257_eng.pdf

3. International Diabetes Federation. IDF Diabetes Atlas, 6th Ed. Basel, Switzerland: International Diabetes Federation. 2013. [cited 2016 Apr. 22]. Available from http://www.idf.org/sites/default/files/DA6_Regional_factsheets_0.pdf

4. Mollentze WF, Levitt NS. Diabetes Mellitus and Impaired Glucose tolerance in South Africa. In: Steyn K, Fourie J, Temple N, editors. Chronic disease of Lifestyle in South Africa since 1995-2005. Medical Research Council - Technical Report. Tygerberg, W Cape: MRC, 2006; p. 109-121.

5. Norman R, Bradshaw D, Shneider M, et al. The South African Comparative Risk Assessment Collaborating Group. A comparative risk assessment for South Africa in 2000: Towards promoting health and preventing disease. S Afr Med J. 2007;97:637-641.

6. Resnikoff S, Pascolini D, Etya'ale $D$, et al. Global data on visual impairment in the year 2002. Bulletin of the WHO. 2004;82(11):844-851.

7. Gotzaridis EV, Markou A, Gregor Z. Management of diabetic retinopathy. An Overview. Hormones. 2004;3(2):92-99.

8. Nicholosi A, Marighi PE, Rizzardi P, Osella A, Miglior S. Prevalence and causes of visual impairment in Italy. Int J epidemiol. 1994;23:359-364.

9. Omolase CO, Adekanle O, Owoe JFA, Omolase BO. Diabetic retinopathy in a Nigerian community. Singapore Med J. 2010;51(1):56-59.

10. Rotchford AP, Rotchford KM. Diabetes in rural South Africa - an assessment of care and complications. S Afr Med J 2002;92:536-541.

11. Read $\mathrm{O}$, Cook $\mathrm{C}$. Retinopathy in diabetic patients evaluated at a primary care clinic in Cape Town. S Afr Med J. 2007;97(10):941-945.

12. Sukha AY, Rubin A. Demographic, medical and visual aspects of Diabetic Retinopathy (DR) and Diabetic Macular Edema (DME) in South African diabetic patients. The South African Optometrist. 2009;68:70-81.

13. Baba D, Krishnan M, Bhaskaran S, Kumar PS, Poovitha R. Prevalence of diabetic Retinopathy and Correlation with Systemic Risk Factors in Type 2 Diabetes Mellitus in a Tertiary Care Hospital. SJAMS. 2015;3(7C):2659-2664.

14. Davis MD, Fisher MR, Gangnon RE. Risk factors for high risk proliferative diabetic retinopathy and severe visual loss: Early Treatment Diabetic Retinopathy Study Report No.18. Invest Ophthalmol Vis Sci. 1998;39:233-252.

15. UK Prospective Diabetes Group. Tight blood pressure control and risk of microvascular and macrovascular complications in type 2 diabetes:UKPDS 38. BMJ. 1998;317(7160):703-713.

16. Chew EY, Klein ML, Ferris FL, et al. Association of elevated serum lipids with retina hard exudates in diabetic retinopathy. Early treatment diabetic retinopathy studies (ETDRS) Report 22. Arch Ophthalmol. 1996;114:1079-1084.

17. Aiello LP, Cahill MT, Wong JS. Systemic considerations in the management of diabetic retinopathy. Am J Ophthalmol. 2001;132(5):760-776.

18. Faruque GM, Ahsan K, Anisuddin AIM. Association of hyperlipedemia with diabetic retinopathy. J Dhaka Med Coll. 2008;17(2):62-66.

19. Jabs DA, Green WR, Fox R, Polk BF, Bartlett JG. Ocular manifestations of acquired immune deficiency syndrome. Ophthalmology. 1989;96(7):1092-1099.

20. Wiwanitkit V. Primary care for diabetes in HIV-infected patients. OJHAS. 2007;6(2).

21. Chakrabarti R, Harper CA, Keeffe JE. Diabetic Retinopathy Management Guidelines. Expert Rev Ophthalmol. 2012;7(5):417-439.

22. Kohner EM, Porta M. Protocols for screening and treatment of diabetic retinopathy in Europe. Eur J. Ophthalmol. 1991;1(1):45-54.

23. Kanski JJ. Clinical Ophthalmology. 6th ed. Retinal Vascular Disease. Netherlands: Elsevier; 2008, p. 566-592. 
24. Murthy GVS, Raman U. Perspectives on primary eye care. Community Eye Health 2009;22(69):10-11.

25. Kanavus P, Van den Aardweg S, Schurer W. Diabetes expenditure, burden of disease and management in $5 \mathrm{EU}$ countries. The London School of Economics Health and Political Science. London, UK. 2012.

26. Wykoff CC, Brown DM. Diabetic Retinopathy: A team approach to Screening, Referral and Treatment. Medscape. 2012. [cited 2013 Feb. 06]. Available from http://www.medscape.com/viewarticle/760431_print

27. Jones SL, Nichols KK. Diabetic eye examination report. Optometry. 2007;78:588-595. http://dx.doi.org/10.1016/j.optm.2007.05.011

28. Fong DS, Aiello L, Gardner TW, et al. Diabetic retinopathy. Diabetes Care 2003;26(Suppl 1):99-102. http://dx.doi.org/10.2337/diacare.26.2007.S99

29. McCarty CA, McKay R, Keeffe JE. Management of diabetic retinopathy by Australian optometrists. Aust N Z J Ophthalmol. 1999;27(6):404-409. http://dx. doi.org/10.1046/j.1440-1606.1999.00263.x

30. McCarthy CA, Taylor KI, Keeffe JE. Management of diabetic retinopathy by general practitioners in Victoria. Clin Exp Ophthalmol. 1987;29:12-16. http://dx.doi. org/10.1046/j.1442-9071.2001.00359.x
31. Raman R, Paul PG, Padmajukumari R, Sharma T. Knowledge and attitude of general practitioners towards diabetic retinopathy practice in South India. Community Eye Health. 2006;19(57):13-14.

32. Dodson PM. Diabetic retinopathy: screening to treatment. Ophthalmology and Heart of England Diabetic Retinopathy Screening Centre, Birmingham, UK. Oxford University Press; 2008.

33. Mash B, Powell D, Du Plessis D, Van Vuuren U, Michalowska M, Levitt N. Screening for diabetic retinopathy in primary care with a mobile fundal camera - Evaluation of a South African pilot project. S Afr Med J. 2007;97(12):1284-1288.

34. Wilkinson CP, Ferris FL, Klein RE, et al. Proposed International DR and DME disease severity scales. Ophthalmology. 2003;110(9):1677-1682. http://dx.doi.org/10. 1016/S0161-6420(03)00475-5

35. Aravind Eye Care System. Disease control, detection within eye care clinics (primary, secondary, tertiary). In: The Right to Sight India Publication. Chapter 3. Guidelines for the Comprehensive Management of Diabetic Retinopathy in India. Geneva: World Health Organization, 2008; p. 15-18. [cited 2011 Aug 29]. Available from www.vision2020india.org/dr_manual.pdf

36. Wiafe B. Who can carry out primary eye care? Community Eye Health 1998;11(26):22-24. 